Aynur Özge

Hakan Kaleagasi

Fazilet Yalçin Tasmertek

\section{Autonomic headache with autonomic seizures: a case report}

Received: 3 April 2006

Accepted in revised form: 18 July 2006

Published online: 25 October 2006
A. Özge $(\bowtie) \cdot H$. Kaleagasi

F. Yalçin Tasmertek

Department of Neurology,

Mersin University Faculty of Medicine,

Mersin 33079, Turkey

e-mail: aozge@mersin.edu.tr

Tel.: +90-324-3374300 (1149)

Fax: +90-324-3374305

\begin{abstract}
The aim of the report is to present a case of an autonomic headache associated with autonomic seizures. A 19-year-old male who had had complex partial seizures for 15 years was admitted with autonomic complaints and left hemicranial headache, independent from seizures, that he had had for 2 years and were provoked by watching television. Brain magnetic resonance imaging showed right hippocampal sclerosis and electroencephalography revealed epileptic activity in right hemispheric areas. Treatment with valproic acid decreased the complaints. The headache did not fulfil
\end{abstract}

the criteria for the diagnosis of trigeminal autonomic cephalalgias, and was different from epileptic headache, which was defined as a pressing type pain felt over the forehead for several minutes to a few hours. Although epileptic headache responds to anti-epileptics and the complaints of the present case decreased with antiepileptics, it has been suggested that the headache could be a nontrigeminal autonomic headache instead of an epileptic headache.

Keywords Headache • Non-trigeminal autonomic cephalalgias • Autonomic seizure $\cdot$ Valproic acid

\section{Introduction}

Although they have a common pathophysiological basis, data about autonomic headaches associated with autonomic seizures are restricted. Autonomic function is often affected during seizures in partial and generalised epilepsies [1]. Headaches with autonomic features, trigeminal autonomic cephalalgias (TACs), are a group of primary headache syndromes characterised by two main clinical characteristics: pain and oculofacial autonomic phenomena [2]. Cluster headache $(\mathrm{CH})$ is characterised by shortlasting, unilateral severe pain attacks, usually located in the orbitotemporal region, which is accompanied by ipsi- lateral autonomic phenomena and/or restlessness or agitation [3]. Activation or inhibition of areas in the central autonomic network can cause cardiovascular, gastrointestinal and cutaneous manifestations. Cranial autonomic involvement in episodic cluster headache $(\mathrm{ECH})$ has been known. Some vegetative symptoms are parts of the diagnostic criteria for ECH and other TACs in the IHS classification. However, there is less information about systemic autonomic impairment. Some authors reported the presence of a parasympathetic, but not sympathetic, involvement in these subjects. Moreover, this impairment seems to be "chronic", as it persists beyond the active period [4].

Cutaneous manifestations of seizures include flushing, sweating, pallor and piloerection [1]. Seizures typically 
activate sympathetic activity, causing increased heart rate and blood pressure, and sinus tachycardia can occur in more than $85 \%$ of complex partial and tonic-clonic seizures $[1,5,6]$. These seizures commonly present as mesial temporal lobe epilepsies (MTLEs), the most widely recognised symptomatic focal epilepsy, arising from the hippocampus, amygdala and parahippocampal gyrus. Almost all patients have complex partial seizures with oral or hand automatisms. Autonomic manifestations include hyperventilation and changes in blood pressure or heart rate, which are more common in MTLEs [7]. Although these seizures have various symptoms, there are no data about headache attacks associated with atypical symptomatic MTLEs. Hemicrania epileptica is a rare known cause of headache and characterised by headache lasting seconds to minutes, with features of migraine, fulfilling the following criteria: the patient has a partial epileptic seizure, headache develops synchronously with the seizure and ipsilateral to the ictal discharge, and headache resolves immediately after the seizure [8,9].

Herein, a case of complex partial seizures associated with hemicranial headache with non-oculofacial autonomic features, and with clear magnetic resonance imaging (MRI) and electroencephalography (EEG) findings is presented and discussed.

\section{Case report}

A 19-year-old male patient with a history of seizures beginning at the age of 4 was admitted with complaints of tachycardia, flushing, epigastric discomfort and left palmar hyperhydrosis, which had followed watching television for 2 years. He had also been complaining of frequent short-lasting headache attacks for 2 years. The seizure frequency was commonly more than 20 per month, generally presenting as sudden onset hyperventilation, dizziness, fainting, palmar hyperhydrosis and flushing in left side of body for 10 or $30 \mathrm{~s}$, and subsequent postictal confusional state for 1 or $2 \mathrm{~min}$. He and his family did not describe any convulsion episode or varying seizure pattern. Sometimes seizures were observed during sleep, but there was no correlation with daily timing or emotional status. No mental or motor developmental abnormality, except generalised anxiety disturbances that did not require any medication, was described in the history. Sometimes vivid hallucinations and taste alterations preceded his seizures. He had complained of frequent headache attacks for two years. The frequency of this severe pressing type headache was 2-4 times/day and 20 days/month, and they lasted for a few hours (not exceeding $3 \mathrm{~h}$ ), especially being clustered at noon time. The headache attacks were not associated with nausea, vomiting or photo/phonophobia, were commonly proceeded by allodynia and trigeminal autonomic signs (hemifacial flushing, eyelid oedema, lacrimation), and did not fulfil the criteria for the strict diagnosis of TACs. We classified the patient's headache as probable TAC (3.4 according to the International Classification of Headache Diagnosis, second edition (ICHD-II)). He did not have any family history of primary headache or epilepsy.

The neurological and cardiac examination, complete blood tests (including biochemical, serological and haematological screening) and the complete analysis of cerebrospinal fluid showed no abnormality. Audiometer and ophthalmologic examinations were also normal. EEG revealed epileptic activity in right hemispheric areas, with slow wave paroxysms and sharp-wave activity on recurrent long-term interictal and ictal bedside portable EEG recordings (Fig. 1). These findings showed a tendency of spreading after seizure records. Brain MRI showed right hippocampal sclerosis (HS) (Fig. 2). Sympathetic skin responses, heart rate variability and routine nerve conduction studies and blood pressure monitoring could not be performed because of technical reasons. The frequency and the severity of seizures with autonomic features and following headache decreased with effective valproic acid (VPA, $1500 \mathrm{mg} /$ day) and lamotrigine

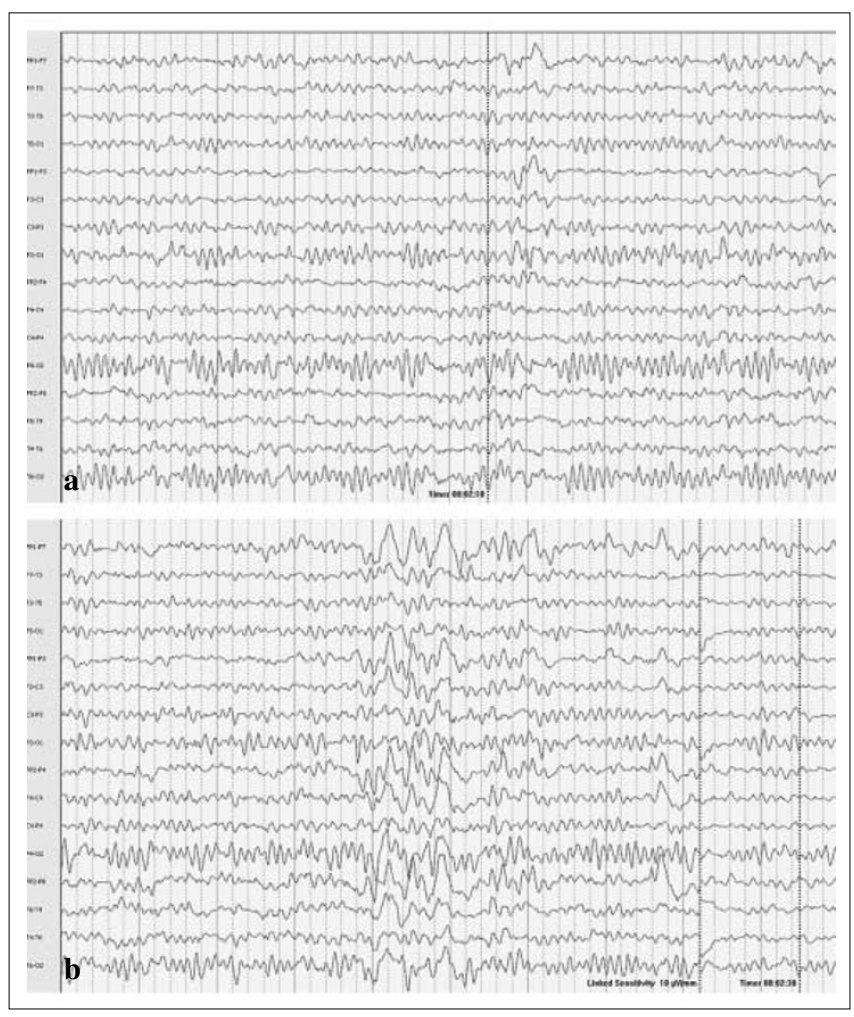

Fig. 1a Right temporoparietal sharp waves of the patient. b The spreading of epileptiform activity during the seizures 

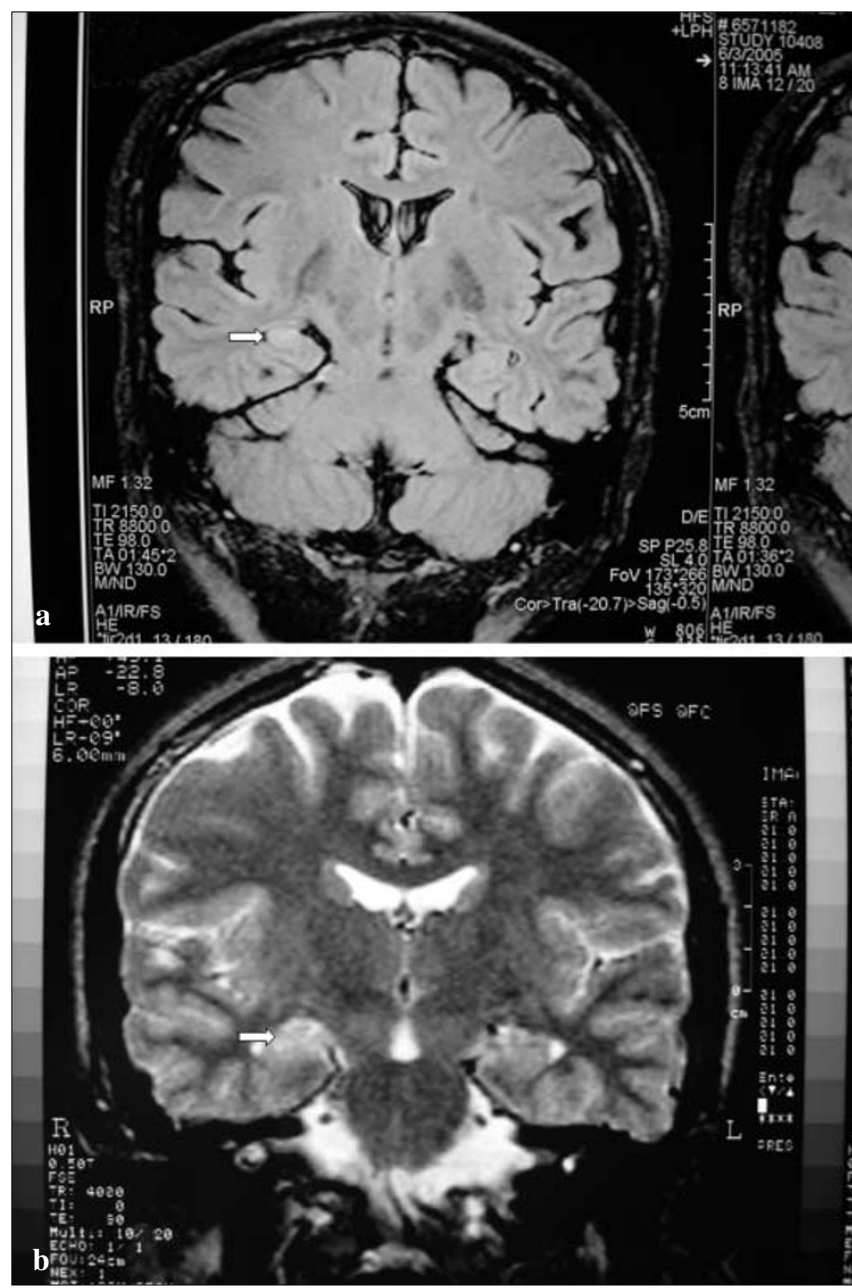

Fig. 2a Right hippocampal sclerosis in T1W coronal section of subject. b Right hippocampal sclerosis in $\mathrm{T} 2 \mathrm{~W}$ coronal section of subject

(125 mg/day) treatment (valproate blood levels were maintained between 50 and $100 \mu \mathrm{g} / \mathrm{ml}$ ) during the followup period. At the last visit after discharge from hospital, he reported rare headache attacks (one or two short-lasting unilateral headache attacks with autonomic features) with 4 seizure attacks, commonly triggered by TV or PC activities. He reported a significant decrease in the duration of postictal confusion and the social impact of the disease after the sixth month of treatment with anti-epileptics.

\section{Discussion}

Headache occurs with seizures as an interictal, preictal, ictal or postictal phenomenon in migraine [8]. However, the present case did not fulfil the migraine criteria according to the ICHD-II criteria [9]. Headache can also occur with seizures as the sole or predominant clinical manifestation [8]. Epileptic headache has been defined as a pressing type pain, which is felt over the forehead contralateral to the epileptic focus for several minutes to a few hours and is sometimes followed by a convulsion. An antiepileptic drug, such as VPA, can be a choice of treatment in such a case, and headache as well as seizure disappears [10]. On the contrary, in the present case, headache was followed by a non-convulsive seizure and did not have the same features. It has been reported that the right hemisphere might predominantly modulate sympathetic tone in human. Sympathetic responses predominate during most seizures, causing tachycardia, tachypnoea, increased blood pressure, pupillary dilatation, diaphoresis and facial flushing [1]. The EEG results also supported this idea, with an epileptic focus in right hemispheric regions in our case. In a recent study, only right temporal seizures were reported to be associated with a significant increase in heart rate whereas left temporal seizures did not cause significant heart-rate changes [11]. These reports can easily explain the autonomic signs accompanying the headache in the present case. Autonomic signs, palmary hyperhydrosis, flushing and tachycardia always occurred with the headache and were triggered by watching television. TACs are reported to be triggered by alcohol, histamine, nitroglycerin, bending head, pressing cervical $2 \mathrm{nd}$, 4th and 5th vertebras, occipital nerve and trigger points on trigeminal nerve area, and neck movements [12]. None of these triggering factors were present in this case. To our knowledge this is the first documented case with autonomic seizures associated with non-trigeminal autonomic cephalalgias.

In their interesting case, Simons et al. reported an adult migraine headache case with complex partial seizures originating from occipital lobe and spreading to temporal lobe because of newly developed HS. Unlike in our case, they reported a history of febrile convulsions, suggested by ictal SPECT findings of occipital hyperperfusion [13]. Like our data, this case supported that the development of HS was associated with the progression of occipital lobe epilepsy towards occipitotemporal lobe epilepsy, also suggesting a complex interaction between an epileptic occipital lobe and HS. As a part of trigeminovascular activation like migraine, TAC and epilepsy suggest the possibility of neuronal channelopathy. This association has been suggested also by a previous detailed experimental study [14].

When the headache and seizure features, EEG findings and significant response to VPA treatment were evaluated, it was thought that the patient's headache was neither TAC nor epileptic headache. As the clinical picture consisted of mainly autonomic features that were triggered by a stimulus, it was thought that the headache might be a nontrigeminal autonomic headache with an accompanying autonomic seizure. 


\section{References}

1. Devinsky O (2004) Effects of seizures on autonomic and cardiovascular function. Epilepsy Curr 4:43-46

2. Leone M, Franzini A, D'Amico D et al (2004) Strategies for the treatment of autonomic trigeminal cephalalgias.

Neurol Sci 25:S167-S170

3. Favier I, Haan J, Ferrari MD (2005) Chronic cluster headache: a review. J Headache Pain 6:3-9

4. Meineri P, Pellegrino G, Rosso MG, Grasso E (2005) Systemic autonomic involvement in episodic cluster headache: a comparison between active and remission periods. $\mathbf{J}$ Headache Pain 6:240-243

5. Opherk C, Coromilas J, Hirsch LJ (2002) Related heart rate and ECG changes in 102 seizures: analysis of influencing factors. Epilepsy Res 52:117-127
6. Leutmezer F, Schernthaner C, Lurger S et al (2003) Electrocardiographic changes at the onset of epileptic seizures. Epilepsia 44:348-354

7. Foldvary N (2001) In: Wyllie E (ed) The treatment of epilepsy: principles, practice, 3rd Edn. Lippincott Williams Wilkins, Philadelphia, pp 467-475

8. Bigal ME, Lipton RB, Cohen J, Silberstein SD (2003) Epilepsy and migraine. Epilepsy Behav 4:S13-S24

9. Headache Classification Subcommittee of the International Headache Society. (2004) The International Classification of Headache Disorders, 2nd edition. Cephalalgia 24[Suppl 1]

10. Sakuta M (2004) Headache originating front-orbital area. Rinsho Shinkeigaku 44:818-819 (Abstract)
11. Saleh Y, Kirchner A, Pauli E et al (2000) Temporallappenepilepsien: Einfluss der Fokusseite auf die autonome Herzfrequenz? Nervenarzt 71:477-480

12. Casucci G (2003) Chronic short-lasting headaches: clinical features and differential diagnosis. Neurol Sci 24:S101-S107

13. Simons PJ, Van Paesschen W, Palmini A et al (2004) The development of hippocampal sclerosis in a patient with occipital lobe epilepsy and migraine. Neurology 62:1024-1025

14. Castle M, Comoli E, Loewy AD (2005) Autonomic brainstem nuclei are linked to the hippocampus. Neuroscience 134:657-669 